\title{
Optimal energy management in series hybrid electric bicycles *
}

\author{
Jacopo Guanetti ${ }^{\text {a }}$, Simone Formentin ${ }^{\text {b }}$, Matteo Corno ${ }^{\text {b }}$, Sergio M. Savaresi ${ }^{\text {b }}$

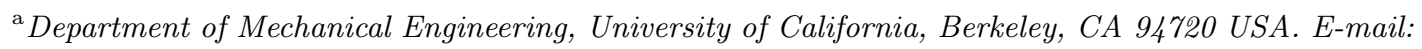 \\ jacopoguanetti@berkeley.edu \\ b Dipartimento di Elettronica, Informazione e Bioingegneria (DEIB), Politecnico di Milano, 20133 Milano, Italy. E-mail: \\ \{simone.formentin, matteo.corno, sergio.savaresi\}@polimi.it
}

\begin{abstract}
Light electric vehicles are a viable way of reducing urban congestion and local pollutant emissions. Electric bicycles combine electrical and human power, hence they represent an appealing class of hybrid vehicles in such a context. Differently from other hybrid vehicles, the energy management of hybrid bicycles is little investigated in the literature. In this work, we study the problem for a series electric-human-powered hybrid architecture, in which there is no direct mechanical link between the pedals and the wheel. We tackle the problem using optimal control principles, aiming to minimize the perceived physical exertion while guaranteeing a predetermined electric range. We build on an approximated solution of the problem and propose a control system that copes with incomplete trip information. In a series of simulation and experimental tests, the proposed strategy approaches the acausal optimum and significantly improves performance if compared to a baseline causal policy; the discussion also encompasses the sensitivity of the results to the requested information.
\end{abstract}

Key words: Optimal Control, human-in-the-loop, Energy Management Systems, Hybrid Electric Bicycles, real-time control.

\section{Introduction}

Electrical Power Assisted Bicycles (EPACs) are meeting growing success in every-day urban usage, due to their convenience in congested areas, to the absence of noise and pollutant emissions and to their efficient use of the energy stored in the battery [2]. EPACs are a class of light Hybrid Electrical Vehicles (HEVs), in that they combine the electrical energy stored in the battery with human power. While hybridization is extensively studied for passenger vehicles and trucks [3-13], energy management of Hybrid Electrical Bicycles (HEBs) has not received as much attention in the literature.

The existing literature is mainly devoted to parallel HEBs, in which the mechanical power at the wheel is supplied by the cyclist and a motor. As in passive bicycles, the cyclist's cadence and the vehicle velocity are kinematically linked. Most products on the market let the cyclist determine the level of assistance, without explicitly accounting for energy efficiency or human dynamics. In $[14,15]$ the optimal pacing to complete a track in minimum time is computed using a model of muscular fatigue; however, the approach is not for online implementation.

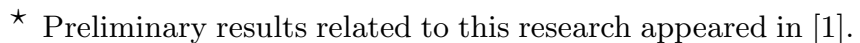
The authors gratefully acknowledge the support of zeHus srl.
}

An online energy management approach for a Full HEB is presented in [16-18]: the algorithm improves the rider's metabolic efficiency, reduces perceived muscular fatigue and sustains the charge of the battery, which needs not be recharged from the grid. The effects on the cyclist's fatigue have been experimentally investigated as well.

Series HEBs lack the mechanical connection between the pedal and the wheel, as the power transmission is purely electric. This in principle lets the cyclist always pedal in optimal conditions, and provides more freedom to the designer to influence the pedal feel and the cyclist's effort $[19,20]$. Possible practical applications include a light Electrical Vehicle (EV) with a human, emission-free range extender, and a moving exercise bike. On another front, the chainless concept opens several control problems, such as ensuring a comfortable feeling at the pedals or managing the dispatch of traction power between the battery and the cyclist.

In this paper, we address the real-time optimal energy management of series HEBs and test it experimentally. Our benchmark is SeNZA, the prototype depicted in Fig. 1 and previously presented in $[21,22]$. The goal is here to minimize an index of perceived exertion and to cope with a constraint on the final state of charge of the battery, to avoid excessive depletion before a charging station is available. 

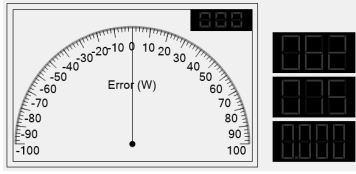

Heart Rate $[\mathrm{bpm}]$

SoC [\%]

Distance $[\mathrm{km}]$

(a) Visual interface for the cyclist.

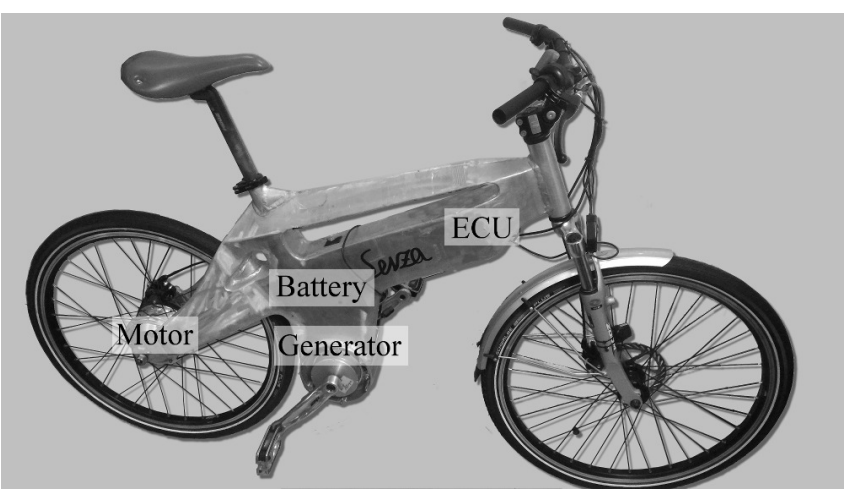

(c) Instrumented prototype of the chainless bicycle SeNZA.

Fig. 1. Some details of the setup employed in the experiments.

With this goal in mind, a review of the physiologic literature suggests to use a cost function based on the cyclist's heart rate; this variable is also easily measurable, therefore model identification and real-time implementation are simplified. In [1], the same setup was the base for an acausal solution study, comparing two approaches based on Pontryagin's Minimum Principle. The first approach was the so-called indirect technique to numerically solve Optimal Control Problems (OCPs) [23]. The second approach was tailored for the series HEB, showing that an approximated technique attains close-to-optimum performance concentrating the knowledge of future cycling conditions in a single tuning parameter. A similar result is known in the literature on the Equivalent Consumption Minimization Strategy (ECMS) [5] for the energy management of HEVs.

In this paper, we build on this approximated approach and propose a real-time strategy, in which the tuning parameter is adapted online to the current cycling conditions. The strategy can use a forecast of the elevation profile, to improve performance and enhance the use of battery charge, especially on hilly routes. While the focus is here on series HEBs, the approach could also be applied to parallel HEBs. An intuitive, simple strategy is also proposed as a baseline. In the proposed simulations, the approach performs closely to the acausal optimum, and outperforms the baseline. A sensitivity study investigates the dependence of these results on the elevation profile. Experimental results are in line with those from simulations, and suggest that the approach can have practical interest.

The paper is structured as follows. The architecture of the chainless bicycle is introduced in Section 2, while the model of the human-electric powertrain is detailed in Section 3. After presenting a baseline strategy in Section 4, the proposed energy management strategy is discussed in Section 5. Section 6 presents the corresponding simula-

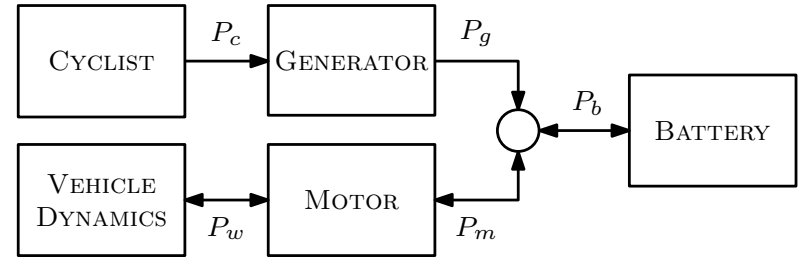

Fig. 2. Conceptual scheme of the series HEB.

tion results, while Section 7 summarizes the experimental results.

\section{System architecture}

The test vehicle, depicted in Fig. 1, is the prototype of SeNZA, a series HEB developed by the mOve research group at the Politecnico di Milano and zeHus srl. For a detailed decription of the prototype the reader is referred to $[21,22]$. In sum, the bicycle is equipped with:

- A $500 \mathrm{~W}$ brushless DC traction motor/generator connected to the rear wheel.

- A $250 \mathrm{~W}$ brushless DC generator linked to the pedals.

- Two custom made Electronic Control Units (ECUs) to control the generator and motor. The generator ECU hosts the energy management system described in this paper. Measurements of motors and battery currents, vehicle velocity and pedal cadence are also available.

- A battery pack, with a Battery Management System (BMS) that performs several safety checks and estimates the state of charge $S o C$ based on current and voltage measurements using a Kalman filter [24].

- Two brake switches, to detect friction braking.

- A CAN bus for the communication between all the ECUs and a data logger.

- A Mio ALPHA Heart Rate Monitor (see Fig. 1) to measure the cyclist's heart rate $H R$.

Fig. 2 depicts the functional representation of the powertrain. Differently from standard HEVs, the prime mover is a cyclist and not a thermal machine. The energy management system splits the motor power $P_{m}$ between battery power $P_{b}$ and generated power $P_{g}$. The motor power $P_{m}$ depends on the wheel power $P_{w}$, while the generated power $P_{g}$ is a function of the output power of the cyclist $P_{c}$. As the arrows in Fig. 2 show, power can only flow from the cyclist to the generator, to either recharge the battery or supply the motor. The motor works as a generator when the wheel power is negative and regenerative braking is performed $[21,22]$.

The control algorithm outputs the optimal amount of electrical power $P_{g}$ that the cyclist should generate. Differently from other HEVs, $P_{g}$ cannot be directly enforced, since it ultimately depends on the cyclist's power output. Hence, a lower layer is introduced to control the human generator in our experiments. The optimal value of $P_{g}$ is sent via CAN bus to a visual interface (see again Fig. 1) that shows to the cyclist the difference between suggested and measured $P_{g}$. Since $P_{g}$ is influenced by both the pedal speed $\omega_{c}$ and the pedal torque $T_{c}$, a PID controller regulates $\omega_{c}$ to a constant value that is comfortable for the 
cyclist. Simultaneously, the cyclist adjusts $T_{c}$ according to the information displayed on the interface, aiming to keep the error between suggested and measured $P_{g}$ close to zero.

To enable a fair comparison between different tests, our experimental setup reproduces a given speed profile. The bicycle is mounted on a roller that replicates the road resistance, and the speed profile is tracked by a speed regulator, also implemented on the generator ECU, that directly controls the torque of the traction motor. For the sake of simplicity, we consider a flat road profile in the experiments; the effect of the elevation profile on performance is studied in simulation.

Finally, in our real-time framework we exploit:

- an estimate of the travel time $\hat{T}$;

- an estimate of the distance to travel $\hat{d}(\hat{T})$;

- an estimate of the road grade profile $\hat{\vartheta}(\hat{d})$;

- a target for the final state of charge $S_{o} C^{o}(\hat{T})$.

The first three pieces of information can be easily obtained with the aid of a navigation system. Setting $\operatorname{SoC}^{o}(\hat{T})$, the user can limit the recharge time spent at destination before being able to travel again.

\section{$3 \quad$ System Modeling}

The supervisor will be designed based on a model of the human-electric hybrid powertrain, that is presented in this section.

Vehicle dynamics The longitudinal dynamics of the bike relate the longitudinal speed $v$ and the road grade $\vartheta$ with the torque $T_{w}$ and speed $\omega_{w}$ of the wheel

$$
M \dot{v}(t)=\frac{T_{w}(t)}{R_{w}}-F_{b}(t)-F_{f}(t), \omega_{w}(t)=\frac{v(t)}{R_{w}},
$$

where $M$ is the mass of the bike and of the cyclist, $R_{w}$ is the wheel radius, $F_{b}$ is the mechanical braking force. The term $F_{f}$ can be detailed as

$$
F_{f}(t)=M g \sin \vartheta(t)+C_{r} M g \cos \vartheta(t)+\frac{1}{2} \rho A C_{x} v(t)^{2},
$$

where $g$ is the gravitational acceleration, $\rho$ is the air density, $A, C_{r}, C_{x}$ are respectively the reference area and the rolling friction and drag coefficients of the bike. Consistent with the above notation, we denote the mechanical power at the wheel by $P_{w}=T_{w} \omega_{w}$.

Traction Motor Since the traction motor is directly connected to the rear wheel, the motor power $P_{m}$ is computed as

$$
P_{m}(t)=P_{w}(t) \eta_{m}^{-\operatorname{sign}\left(T_{w}(t)\right)},
$$

where the efficiency $\eta_{m}\left(T_{w}, \omega_{w}\right)$ depends on the mechanical operating point.

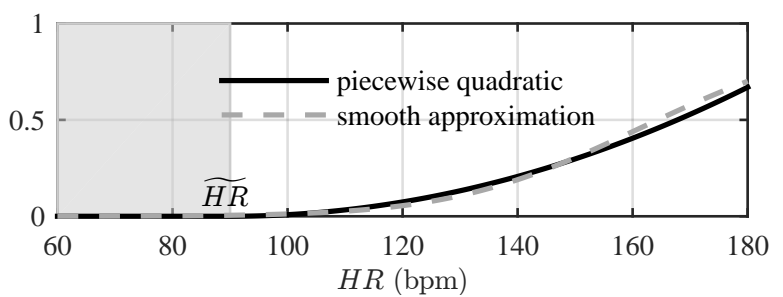

Fig. 3. Two choices for $H R$ weighting: $l^{*}$ in (5) and $l$ in (6).

Power Link The power at the traction motor can be supplied by the battery and the electric generator $P_{m}(t)=P_{b}(t)+P_{g}(t)$, where $P_{b}$ is the battery power and $P_{g}$ is the electrical power generated by the cyclist.

Battery The battery power can be computed as $P_{b}(t)=v_{b}(t) i_{b}(t)$, where $v_{b}$ is the voltage across the battery pack and $i_{b}$ is the current (positive in discharge). The equivalent circuit encompasses a voltage generator and a resistor. The former outputs the open circuit voltage $v_{o c}$ that is measured when the battery is disconnected from any load. The resistor $R_{b}$ accounts for Joule losses due to non-ideal electro-chemical conversion. The equation for battery voltage is therefore $v_{b}(t)=v_{o c}(t)-R_{b} i_{b}(t)$, where $R_{b}$ is constant and $v_{o c}$ is an affine function of the battery state of charge $S o C[6], v_{o c}(t)=A_{b} S o C(t)+B_{b}$. The parameters $A_{b}, B_{b}$ are found experimentally.

In the model, the state of charge of the battery $S o C \in$ $[0,1]$ is defined $[6]$ based on the battery capacity $Q_{b}$

$$
\operatorname{So} C(t)=-\frac{i_{b}(t)}{Q_{b}}
$$

Human physiology and cyclist modeling The physiological effects of cycling have been fairly studied in the literature, for instance in [25-29]. Most models, however, are not developed for control purposes and rely on sophisticated equipment, experiments and tuning that make them unsuitable for real-time applications.

Our focus is on the penalization of physical exertion, which has been related e.g. to aerobic and anaerobic reserves $[30,31]$, to muscular fatigue $[14,15]$ and to heart rate [32]. Since the heart rate $(H R)$ is easy to measure, real-time applications employing this variable are envisaged. In particular, in [32] it is shown that different exertion scales can be defined, each one having a different functional dependence on the $H R$.

A possible choice is the piecewise-quadratic running cost

$$
l^{*}(t)=\left\{\begin{array}{ll}
0, & H R(t)<\widetilde{H R} \\
\nu(H R(t)-\widetilde{H R})^{2} d t, & H R(t)>\widetilde{H R}
\end{array} .\right.
$$

Ideally, a very high $H R$ is penalized more because it is a reliable indicator of intense activity; below a threshold $\widetilde{H R}$, no penalization is introduced, since small deviations 
may be due to other reasons than physical activity. However, a non-smooth running cost complicates the mathematical analysis; thus, a smooth running cost is adopted

$$
l(t)=\nu \frac{\kappa_{1}^{2}}{\left(\kappa_{2}+e^{-\kappa_{3} H R(t)+\kappa_{4}}\right)^{2}} .
$$

A comparison between the two choices $l^{*}$ and $l$ is shown in Fig. 3.

For a control oriented model of the $H R$ as a function of the cyclist power $P_{c}$ we refer to [33] and the references quoted therein. In [33] a dynamic model is proposed and experimentally validated for a cyclist riding a bicycle equipped with a Continuously Variable Transmission (CVT). The following model is found to be a reasonable compromise between accuracy and complexity:

$$
H R=H R_{0}+G_{c}(s) P_{c}
$$

where $H R_{0}$ is the base $H R$ and $G_{c}$ is the transfer function

$$
G_{c}(s)=\frac{\mu_{c}}{\left(1+s T_{1}\right)\left(1+s T_{2}\right)}
$$

According to this model, deviations of the $H R$ from its base value are proportional to the output power in steady state (with a factor $\mu_{c}$ ); this is consistent with energetic models and can be related to aerobic mechanisms. Transients - involving anaerobic mechanisms - are modeled through two time constants $T_{1}, T_{2}$.

The mechanical power produced by the cyclist is converted into electrical power by means of an electric machine with efficiency $\eta_{g}, P_{g}(t)=P_{c}(t) \eta_{g}$.

\section{Baseline Strategy}

Before introducing the proposed technique, a baseline strategy is presented. The latter only employs a $S o C$ feedback, basic route information and a simple regulation architecture. It is used in the next sections as a real-world benchmark for the proposed control approach. Clearly, different baseline policies can be defined for other purposes: due to lack of space, this task is left to future research.

We consider as a baseline strategy a PI controller that employs only the $S o C$ measurement to track a reference signal $S o C^{o}$. While good tracking performance facilitates the fulfillment of the $S o C$ constraints, it also requires higher levels of power generation to the cyclist. Tuning is therefore a compromise between these two conflicting objectives. We found an initial tuning linearizing the battery model (with input $P_{b}$ and output $S o C$ ) and setting the closed loop bandwidth to about $0.01 \mathrm{rad} / \mathrm{s}$. The final tuning (proportional gain $3.2 \times 10^{4}$, integral gain 0.77 ) was then obtained with the aid of simulations on the cycling profiles analyzed in the remainder of the paper. The implemented power split logic also considers the case when

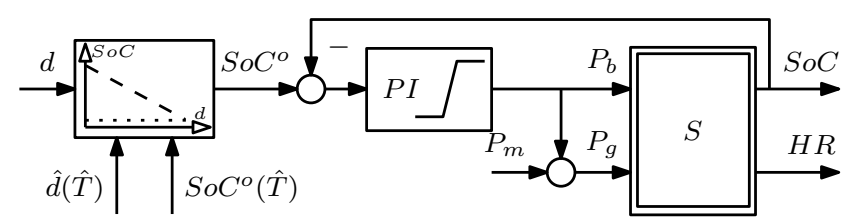

Fig. 4. Block diagram of the baseline strategy. $d$ and $\hat{d}(\hat{T})$ indicate elapsed and estimated total distance, respectively.

the cyclist does not supply the requested power: the surplus power can still be supplied by the battery, unless the legislation constraints are exceeded $(250 \mathrm{~W}$ in steady state for this work). In the latter case, less power than required is supplied to the motor.

The reference $S o C^{o}$ influences the cyclist's workload distribution along the trip. If no information is available (neither on the target $S_{o} C^{o}(\hat{T})$ at destination, nor on the distance to travel $\hat{d}(\hat{T}))$, the reference $S o C^{o}$ is a ramp decreasing from the initial $S o C$ to the minimum acceptable SoC and a constant afterwards; the slope of the ramp is decided at the design stage and clearly affects the overall performance. A more favorable situation is considered here, i.e. the target $S_{o} C^{o}(\hat{T})$ and the estimate $\hat{d}(\hat{T})$ are known in advance. Hence, the reference $S o C^{o}$ is simply generated as a ramp decreasing linearly with distance, from the initial $S o C$ to the target $S_{o} C^{o}(\hat{T})$.

\section{Real-time Control}

In this section we propose a real-time control strategy. We first summarize the acausal optimal control policy described in [1] that we use in the remainder of the paper (we refer to the original work for details); we then describe the proposed real-time control framework in detail.

\subsection{Optimal and approximated acausal policies}

In [1], the energy management of the series HEB is formulated as an OCP with time horizon $T$. The output power of the generator is the control variable $u=P_{g}$, the traction motor power is an exogenous input $w=P_{m}$, and the state variables are (i) the $S o C$ of the battery $x_{1}=S o C$, (ii) the deviation of the $H R$ with respect to the base value $x_{2}=H R-H R_{0}$ and (iii) its derivative $x_{3}=\dot{H R}$, as defined by (7), (8). The control constraints $\underline{u}(x, w)<u(t)<\bar{u}(x, w)$ depend on the current values of the states and of the exogenous input, as well as on static bounds on $P_{g}$ and $P_{b}\left(\underline{P}_{g}, \bar{P}_{g}, \underline{P}_{b}, \bar{P}_{b}\right)$. The $S o C$ is constrained to the set $[\underline{S o C}, \overline{S o C}]$, and a soft terminal constraint is set to bring the terminal $S o C(T)$ to a target value $S_{o} C^{o}(T)$.

The main analytical tool in [1] is Pontryagin's Minimum Principle, which has also been used for HEV energy management (see e.g. the ECMS [5,7] and the least costly approach [34-37]). The Minimum Principle gives the analytical expressions of the Hamiltonian $H$, of the adjoint states derivatives $\dot{p}_{1}, \dot{p}_{2}, \dot{p}_{3}$ and of their boundary conditions $p_{1}(T), p_{2}(T), p_{3}(T)$. An optimal control policy must 


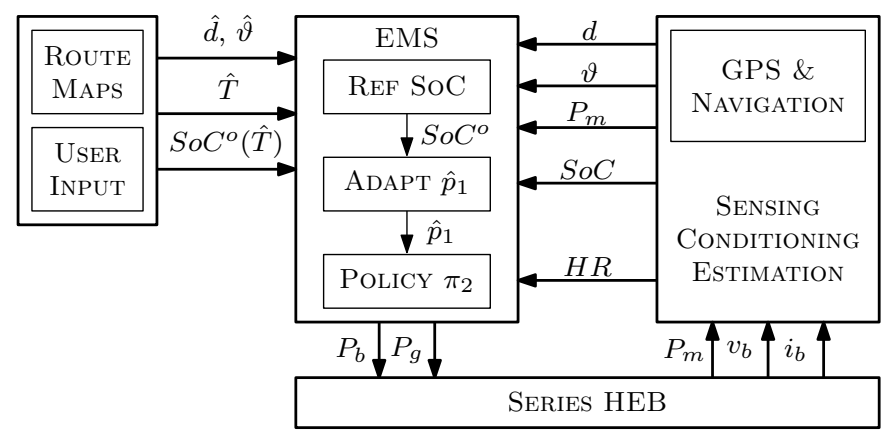

Fig. 5. Proposed control architecture. $d$ and $\hat{d}$ indicate elapsed distance and estimated total distance, respectively.

satisfy

$$
\pi_{1}(t)=\arg \min _{u(t) \in \mathcal{U}}\{H(t)\}, \forall 0 \leq t \leq T
$$

and the optimal trajectories are found solving numerically the resulting two point Boundary Value Problem (BVP). Policy $\pi_{1}$ will be used as the acausal benchmark in the remainder of the paper.

A suboptimal policy $\pi_{2}$ is also defined in [1], approximating (under some hypotheses detailed therein) the derivatives of the adjoint states to zero $\dot{p}_{1}=\dot{p}_{2}=\dot{p}_{3} \approx 0$. Policy $\pi_{2}$ is still based on Hamiltonian minimization; however, the adjoint states are now constant and a simpler technique can be used instead of solving the BVP. Global performance is found to depend on the (constant) value of the first adjoint state $p_{1}$, which affects the final value of the $S o C$. In the acausal setting considered in [1], the optimal value of $p_{1}$ can be found iteratively; the resulting performance is close to the optimum defined by $\pi_{1}$.

In the real-time setting considered here, future route information is incomplete and uncertain. Nonetheless, policy $\pi_{2}$ is a good starting point for real-time control. The main issue for real-time implementation is to exploit the available information to optimally adapt $\hat{p}_{1}$. In the literature on energy management of HEVs and plug-in HEVs, causal approaches like the Telemetry-based ECMS (TECMS) [8] and the Adaptive ECMS (A-ECMS) [9] mitigate the uncertainty adapting the adjoint state in realtime. In several literature contributions [10-13,35,38-40], this adaptation uses past data and limited information on the future driving schedule. Remarkably, in plug-in HEVs and extended range EVs this enables the optimal depletion of battery charge.

The causal implementation presented in this section is depicted in Fig. 5. The power split is based on policy $\pi_{2}$; since the optimal tuning of $p_{1}$ requires the full knowledge of future driving conditions, an estimate $\hat{p}_{1}$ is adapted according to a causal law, based on the error between the current $S o C$ and its reference value $S o C^{o}$. The causal law, presented in the next sub-section, is time invariant, but can be tuned at the beginning of the trip. The following sub-section shows how the reference $S o C^{\circ}$ is generated at the beginning of the trip.

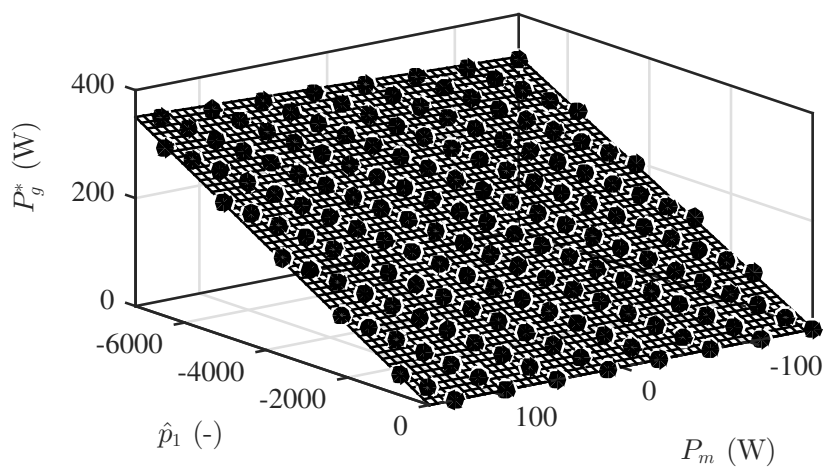

Fig. 6. Fitting of the optimal closed loop power $P_{g}^{*}$ in steady state (black points) with the proposed relationship (10).

\section{2 $\hat{p}_{1}$ causal adaptation law}

A PI law based on $S o C$ tracking error is used here to adapt $\hat{p}_{1}$ to the actual driving conditions, in order to guarantee that the $S o C$ at the end of the trip is close to the specified target. This law is derived based on a simplified model of the system when policy $\pi_{2}$ is applied. The optimal closed loop power $P_{g}^{*}$ for a static exogenous input $P_{m}$ is approximated with a relationship of the form

$$
P_{g}^{*}=\hat{p}_{1}\left(\varphi_{1} P_{m}+\varphi_{2}\right)=\hat{p}_{1} \phi\left(P_{m}\right)
$$

where $\varphi_{1}$ and $\varphi_{2}$ are fitting parameters. Fig. 6 compares the fitted surface to the optimal values of $P_{g}^{*}$, corresponding to constant values of $P_{m}$ (on the $x$ axis) as a function of the tuning parameter $\hat{p}_{1}$ (on the $y$ axis). Long-term predictions of $P_{m}$ based on route forecasts are inherently quasi-static, because fast transients are mostly due to unpredictable phenomena, such as traffic or human behavior. Therefore, the approximated model (10) seems a reasonable way to describe the system behavior under policy $\pi_{2}$ on slow time scales.

Taking the approximation $P_{b} \approx i_{b} v_{o c}$ one gets

$$
\hat{p}_{1} \approx \frac{P_{g}^{*}}{\phi\left(P_{m}\right)}=\frac{P_{m}-P_{b}}{\phi\left(P_{m}\right)}=\frac{P_{m}}{\phi\left(P_{m}\right)}+\frac{Q_{b} v_{o c}}{\phi\left(P_{m}\right)} S \dot{o} C
$$

After applying the Laplace transform, a PI law can be tuned based on the approximated transfer function from the adjoint state $\hat{p}_{1}$ to the battery $S o C$

$$
\frac{S o C(s)}{\hat{p}_{1}(s)}=-\frac{\phi\left(\hat{P}_{m}\right)}{s Q_{b} v_{o c}}
$$

where $\hat{P}_{m}$ is a prediction of the average motor power in the trip. A block diagram of the approximated system considered for the tuning is depicted in Fig. 7. A PI controller with proportional gain $K_{p}$ and integral time $T_{i}$ is tuned to achieve a closed loop cutoff frequency $\omega_{c}$ in the $S o C$ reference tracking. Assuming to choose $T_{i} \gg 1 / \omega_{c}$, in a neighborhood of the cutoff frequency the loop trans- 


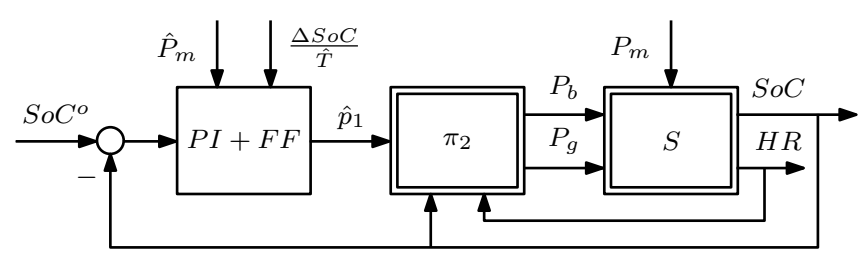

Fig. 7. Block diagram of policy $\pi_{2}$ with causal adaptation of the adjoint state $p_{1}$. Block $S$ indicates the battery and the human-electric generator.

fer function is approximated as

$$
L(s) \approx-\frac{\phi\left(\hat{P}_{m}\right) K_{p}}{s Q_{b} v_{o c}},
$$

which can be used to tune the proportional gain based on the desired cutoff frequency

$$
K_{p}=\omega_{c} \frac{Q_{b} v_{o c}}{\phi\left(\hat{P}_{m}\right)} .
$$

In addition to the PI law, it is beneficial to add a constant term to the estimate

$$
\hat{p}_{1}^{F F}=\frac{\hat{P}_{m}}{\phi\left(\hat{P}_{m}\right)}+\frac{Q_{b} v_{o c}}{\phi\left(\hat{P}_{m}\right)} \frac{\Delta S o C}{\hat{T}},
$$

which comes directly from (11) if the estimates of the motor power $\hat{P}_{m}$, of the trip duration $\hat{T}$ and of the battery discharge $\Delta S o C=S o C^{o}(\hat{T})-S o C(0)$ are used. In Section 2, we assumed to know the latter two quantities, as well as an estimate of the overall distance $\hat{d}$. The average speed for the trip can then be estimated, and using the vehicle model in Section 3 the average $\hat{P}_{m}$ is found. The estimate of $\hat{P}_{m}$ can be refined using the knowledge of the elevation profile, if available. The resulting adaptation scheme for $\hat{p}_{1}$ is represented in Fig. 7 .

\subsection{SoC reference generation}

The real-time strategy is now completed with a generator of the reference $S o C^{o}$. This can be done in a basic way as in the baseline strategy, where the reference $S o C^{o}$ decreases linearly with the traveled distance, from the initial $S o C$ to the target $S_{o} C^{o}(\hat{T})$. In this case, the only a-priori information needed is the overall trip distance.

If more information on the route is available, it can be used to generate the reference $S o C^{o}$ in a more accurate way. This is beneficial for instance when riding on hilly routes, where the optimal policy can yield very long segments of battery depletion during uphills and of regenerative braking during downhills; in such a case, the optimal $S o C$ trend significantly deviates from a simple decreasing ramp, and a suboptimal policy can violate the $S o C$ operating constraints, as well as not meet the target $S o C$.

In the simulations presented in this work, the reference generation procedure is run at the beginning of the trip and can be summarized as follows:
Table 1

Model and control parameters

\begin{tabular}{clll}
\hline$M$ & bicycle and cyclist mass & $\mathrm{kg}$ & 100 \\
$R_{w}$ & wheel radius & $\mathrm{m}$ & 0.35 \\
$C_{r}$ & bicycle roll coefficient & - & 0.0036 \\
$C_{x}$ & bicycle drag coefficient & - & 0.9 \\
$\rho_{a}$ & air density & $\mathrm{kg} / \mathrm{m}^{3}$ & 1.18 \\
$A$ & bicycle and cyclist reference area & $\mathrm{m}^{2}$ & 0.4 \\
\hline$A_{b}$ & battery $v_{o c}$ parameter & $\mathrm{V}$ & 5 \\
$B_{b}$ & battery $v_{o c}$ parameter & $\mathrm{V}$ & 37 \\
$Q_{b}$ & battery nominal capacity & $\mathrm{A} \mathrm{h}$ & 10 \\
$R_{b}$ & battery internal resistance & $\Omega$ & 0.25 \\
$S o C_{0}$ & battery initial state of charge & $\%$ & 75 \\
$\overline{S o C}$ & maximum battery state of charge & $\%$ & 90 \\
$S_{o} C$ & minimum battery state of charge & $\%$ & 20 \\
$\bar{P}_{b}$ & maximum battery power & $\mathrm{W}$ & 250 \\
$\underline{P}_{b}$ & minimum battery power & $\mathrm{W}$ & -1000 \\
\hline$\eta_{g}$ & generator efficiency & - & 0.8 \\
$H R_{0}$ & cyclist base heart rate & $\mathrm{bpm}$ & 60 \\
$\mu_{c}$ & cyclist heart rate parameter & $\mathrm{bpm} / \mathrm{W}$ & 0.3 \\
$T_{1}$ & cyclist heart rate parameter & $\mathrm{s}$ & 22 \\
$T_{2}$ & cyclist heart rate parameter & $\mathrm{s}$ & 55 \\
$\bar{P}_{g}$ & maximum generated power & $\mathrm{W}$ & 1000 \\
$\underline{P}_{g}$ & minimum generated power & $\mathrm{W}$ & 0 \\
\hline
\end{tabular}

- A-priori information, i.e. target $S_{o} C^{o}(\hat{T})$ and desired/expected trip duration $\hat{T}$, is collected from the user.

- Available information on the track, i.e. distance and elevation profile, is collected from a navigation system (here we use Google Maps API).

- Speed and slope profiles are estimated with a heuristic, ensuring that the trip distance $\hat{d}$ is covered in the expected duration $\hat{T}$. A constant wheel power $P_{w}$ is pursued on flat and uphill segments; in other words, in steady state the speed estimate is a function of slope only. Speed transients are done at constant acceleration and deceleration rates, for simplicity. Moreover, during downhill segments the speed is saturated to $25 \mathrm{~km} / \mathrm{h}$ and the wheel power $P_{w}$ is computed accordingly.

- The motor power is estimated simulating the models of the vehicle body dynamics and of the traction motor, presented in Section 3.

- The optimal power generation policy is estimated. The average generated power $P_{g}$ is assumed to equal the average motor power $P_{m}$ minus the constant value of battery power $P_{b}$ such that the target $S o C$ is achieved at the end of the trip. In this computation, $P_{b}$ is saturated to $250 \mathrm{~W}$ due to legislation limits; the average cyclist load is lowered to compensate the additional load when battery is saturated.

- The reference $S o C^{o}$ signal is computed based on the estimated profile of the battery power

$$
S o C^{o}(t)=S o C(0)-\frac{1}{Q_{b} v_{o c}} \int_{0}^{t} P_{b}(\tau) d \tau \text {. }
$$

\section{Simulation Results}

In this section, we present a simulation study to compare the performance of:

- policy $\pi_{1}$, the acausal strategy presented in Subsec- 
Table 2

Parameters of the cycling tracks.

\begin{tabular}{|c|c|c|c|c|c|}
\hline Track & & $\mathcal{T}_{1}$ & $\mathcal{T}_{2}$ & $\mathcal{T}_{3}$ & $\mathcal{T}_{4}$ \\
\hline Length & $\mathrm{km}$ & 18.2 & 6.6 & 42 & 3.3 \\
\hline Max speed & $\mathrm{km} / \mathrm{h}$ & 25 & 25 & 25 & 22 \\
\hline Average speed & $\mathrm{km} / \mathrm{h}$ & 10 & 16 & 16 & 11 \\
\hline Duration & $\min$ & 109 & 25 & 150 & 15 \\
\hline Max uphill slope & $\%$ & 18 & 15 & 15 & 0 \\
\hline Min downhill slope & $\%$ & 14 & 11 & 11 & 0 \\
\hline Altitude variation & $\mathrm{m}$ & 210 & 70 & 70 & 0 \\
\hline
\end{tabular}

tion 5.1 representing the optimum;

- $\operatorname{policy} \pi_{s}$, the causal strategy proposed in Section 5 and summarized in Fig. 5;

- policy $\pi_{b}$, the baseline strategy presented in Section 4 .

In particular, we inspect the trajectories of the most significant variables and compare the relative cost with respect to policy $\pi_{b}$

$$
\Phi(H R) \triangleq 100 \frac{\left.\int_{0}^{T} l(t) d t\right|_{\pi_{i}}}{\left.\int_{0}^{T} l(t) d t\right|_{\pi_{b}}}
$$

and the peak value of the $H R$

$$
\|H R\|_{\infty} \triangleq \sup _{0 \leq t \leq T} H R(t)
$$

Another index is defined as the error in battery internal energy at the end of the trip, normalized to the motor energy

$$
\Gamma_{S o C}=100 \frac{v_{o c} Q_{b}}{\int_{0}^{T} P_{m}(t) d t}\left(S o C(T)-S o C^{o}(T)\right) .
$$

Hence, the deviation from the final $S o C$ target is weighted according to the total energy demanded during the trip. The model described in Section 3 is implemented in Simulink using the parameters summarized in Table 1.

Several real-world cycling profiles are compared: their main parameters are summarized in Table 2 . Track $\mathcal{T}_{1}$ shows significant distance, altitude variation and peaks in the road slope; the intensity is partially mitigated by the low average speed. Track $\mathcal{T}_{2}$ combines altitude variations, peak slopes and average speed; it represents a brief but intense workout. Track $\mathcal{T}_{3}$ covers a long distance at relatively high average speed, has significant altitude variations, and represents a long workout of an amateur cyclist.

\subsection{Cycling Track $\mathcal{T}_{1}$}

The three policies are compared in Fig. 8 for the case of the cycling track $\mathcal{T}_{1}$; the terminal target $S o C^{o}(T)$ is initially set to $55 \%$, which is an intermediate value between charge sustenance $(75 \%)$ and maximum assistance to the cyclist $(37.2 \%)$.

The comparisons in Fig. 8 show some deviations of the proposed strategy $\pi_{s}$ from the benchmark policy $\pi_{1}$. In
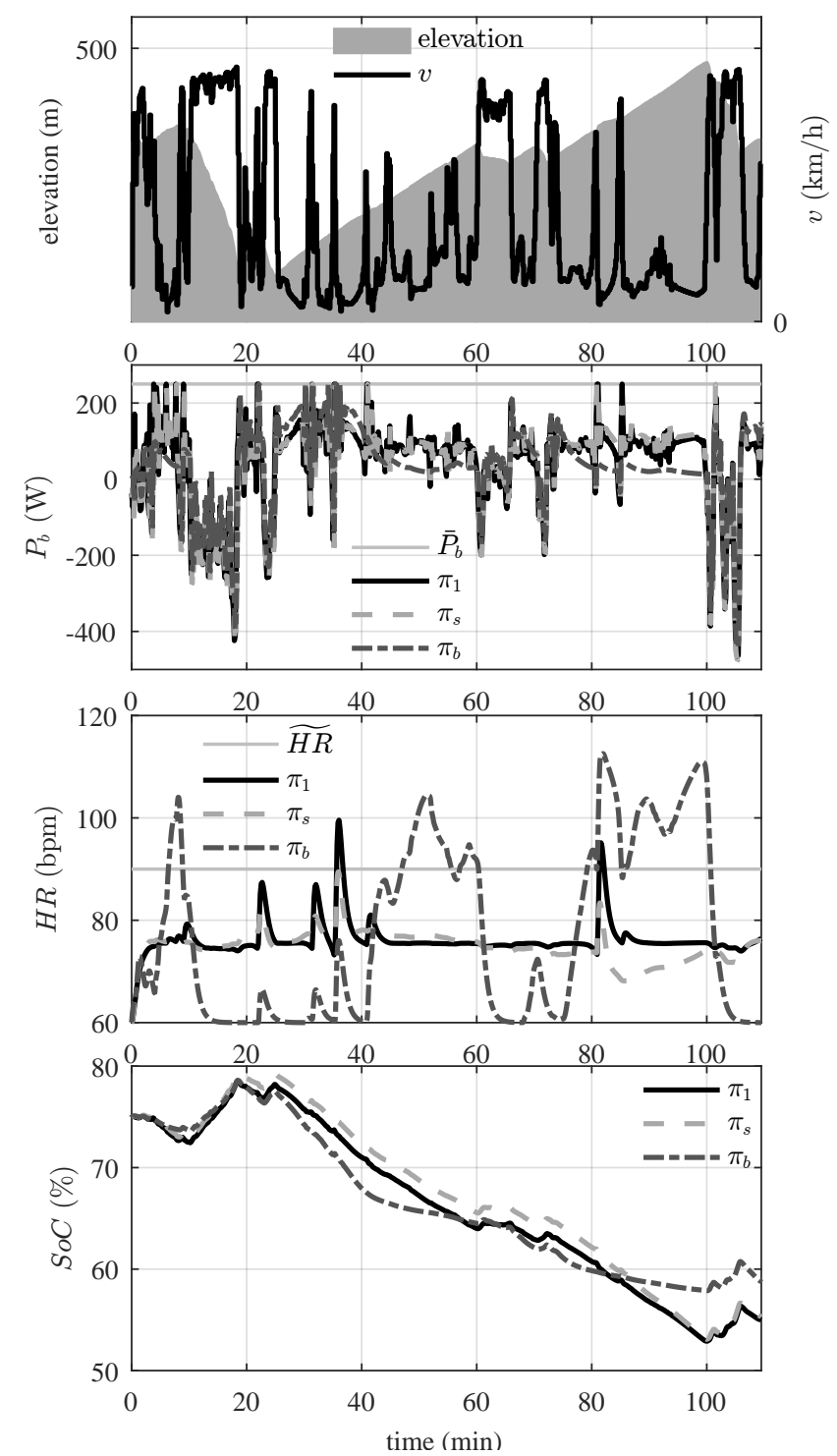

Fig. 8. Simulation of policies $\pi_{1}, \pi_{s}, \pi_{b}$ on Track $\mathcal{T}_{1}$ : speed and elevation, battery power $P_{b}$, heart rate $H R$, state of charge SoC.

the long uphill phase, the $S o C$ with policy $\pi_{s}$ is slightly higher than with policy $\pi_{1}$; after reaching a maximum around minute 60 , the difference decreases and the two policies attain the same final $S o C$. The deviation in the $S o C$ is reflected in a deviation in the $H R$ : from minute 40 to minute 60 , policy $\pi_{s}$ yields a (slightly) higher $H R$ than $\pi_{1}$; conversely, after minute 60 policy $\pi_{s}$ increases the

Table 3

Real-time control: simulation results on Track $\mathcal{T}_{1}$.

\begin{tabular}{ccccc}
\hline$S_{S o C^{o}(T)(\%)}$ & & $\Phi(H R)(\%)$ & $\|H R\|_{\infty}(\mathrm{bpm})$ & $\Gamma_{S o C}(\%)$ \\
\hline \multirow{3}{*}{37.2} & $\pi_{1}$ & - & 76 & 0 \\
& $\pi_{s}$ & - & 76 & 0 \\
& $\pi_{b}$ & - & 79 & -2 \\
\hline \multirow{3}{*}{55} & $\pi_{1}$ & 0 & 87 & 0 \\
& $\pi_{s}$ & 0 & 89 & -1 \\
& $\pi_{b}$ & 100 & 113 & -10 \\
\hline \multirow{2}{*}{75} & $\pi_{1}$ & 0 & 99 & 0 \\
& $\pi_{s}$ & 0 & 102 & 2 \\
& $\pi_{b}$ & 100 & 125 & -3 \\
\hline
\end{tabular}


battery usage, thus reducing the $H R$ and the deviation of the $S o C$ from the optimal trend.

Table 3 summarizes the performance indexes, showing that there is no significant difference in the cumulated cost: both $\pi_{1}$ and $\pi_{s}$ keep the $H R$ below $\widetilde{H R}$, aside from two brief events; the peak $H R$ is only $2 \mathrm{bpm}$ higher with policy $\pi_{s}$. Conversely, the baseline policy $\pi_{b}$ leads to increased cumulated cost and to a peak $H R 26 \mathrm{bpm}$ higher than the optimum. This is also apparent from Fig. 8: the $H R$ suggests that the load on the cyclist is not equally distributed along the route, which brings the $H R$ well over $\widetilde{H R}$ during the long uphill segments.

Table 3 summarizes also the performance for other $S o C$ scenarios, namely $\operatorname{SoC}^{o}(T)=37.2 \%$ and $S_{o} C^{o}(T)=$ $75 \%$. Although in both cases the baseline policy $\pi_{b}$ achieves a final $S o C$ closer to the target (the index $\Gamma_{S o C}$ is smaller than in the other case), the performance in terms of $H R$ is below that of policy $\pi_{s}$. This is especially true in the charge sustaining case: the same comments as before apply and the only relevant difference is that - due to the higher load on the cyclist - the peak $H R$ is higher than $\widetilde{H R}$ also for policy $\pi_{s}$.

\subsection{Cycling Track $\mathcal{T}_{2}$}

The recorded speed and elevation profiles for this route are shown in Fig. 9. For this scenario, the feasible target set is found to be $67 \% \leq S o C^{\circ}(T) \leq 75 \%$; the intermediate case of $S_{o} C^{o}(T)=71 \%$ is analyzed first. Both speed and elevation profiles are quite harsh and their combination make the cycle demanding, as the $H R$ profiles in Fig. 9 suggest. During the initial descent - until minute 10 approximately - the optimal policy $\pi_{1}$ maintains the $S o C$ to the initial level, with little deviations due to local changes of slope and speed. In a specular way, the cyclist $H R$ settles at about $80 \mathrm{bpm}$ after an initial transient and again with some deviations due to local events. The uphill segment - until minute 17 - causes the steep drop of the $S o C$, which falls down to the final level. Notice that in this phase the battery is operating almost constantly at maximum power, meaning that the discharge rate and the assistance to the cyclist are maximum. This is the reason why the $H R$ is so irregular during this segment, with a peak of about $170 \mathrm{bpm}$ : the cyclist has to supply all the excess power required to follow the cycling profile. The final segment has a quite steep descent and a brief uphill in the very last portion of the route. It is observed

Table 4

Real-time control: simulation results on Track $\mathcal{T}_{2}$.

\begin{tabular}{crrcc}
\hline$S_{0} C^{o}(T)(\%)$ & & $\Phi(H R)(\%)$ & $\|H R\|_{\infty}(\mathrm{bpm})$ & $\Gamma_{S o C}(\%)$ \\
\hline \multirow{2}{*}{67} & $\pi_{1}$ & 99 & 166 & 0 \\
& $\pi_{s}$ & 100 & 167 & 0 \\
& $\pi_{b}$ & 100 & 167 & -1 \\
\hline \multirow{2}{*}{71} & $\pi_{1}$ & 19 & 167 & 0 \\
& $\pi_{s}$ & 23 & 174 & -3 \\
& $\pi_{b}$ & 100 & 238 & -7 \\
\hline \multirow{2}{*}{75} & $\pi_{1}$ & 22 & 168 & 0 \\
& $\pi_{s}$ & 23 & 182 & 6 \\
& $\pi_{b}$ & 100 & 248 & -1 \\
\hline
\end{tabular}
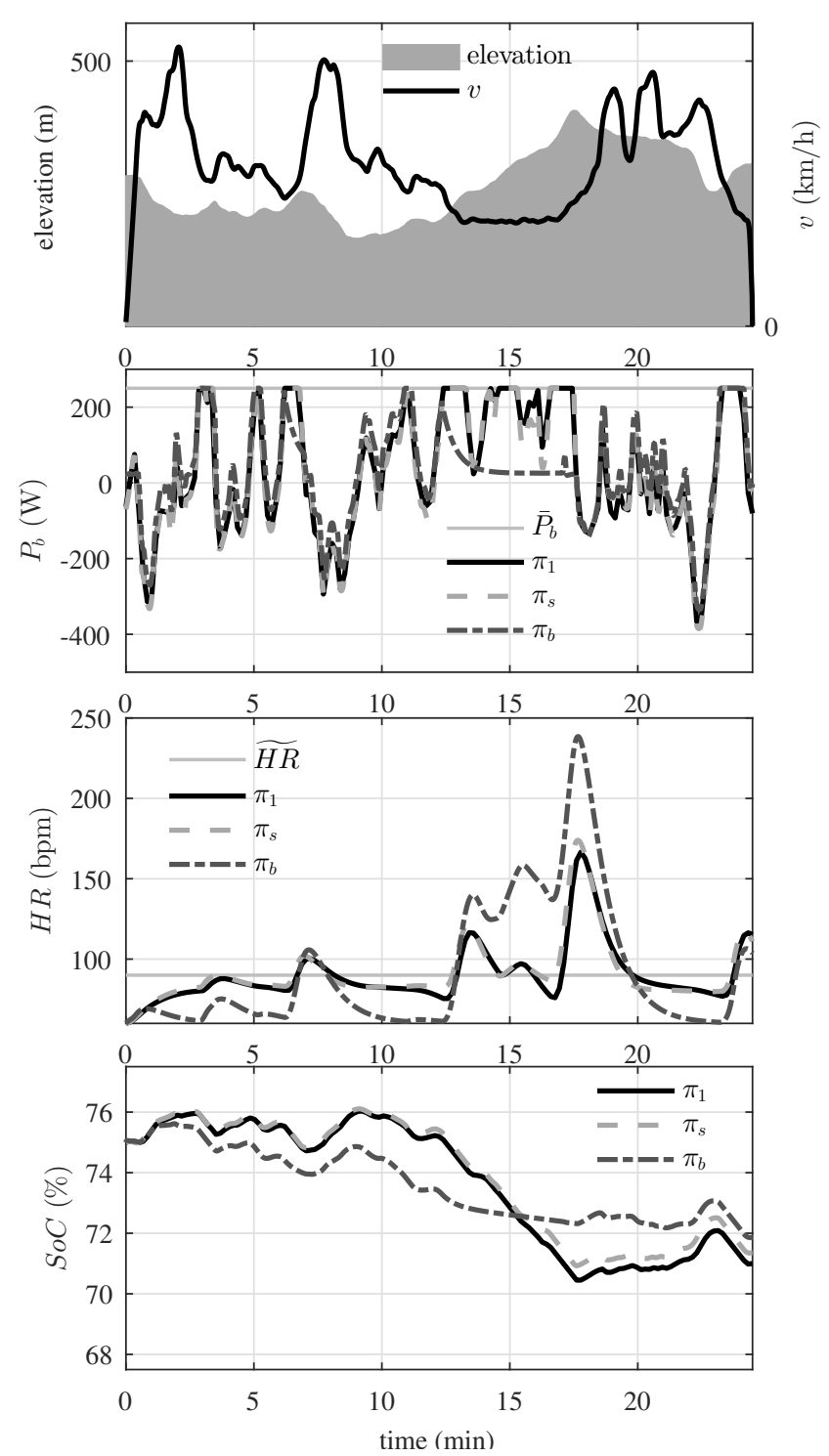

Fig. 9. Simulation of policies $\pi_{1}, \pi_{s}, \pi_{b}$ on Track $\mathcal{T}_{2}$ : speed and elevation, battery power $P_{b}$, heart rate $H R$, state of charge SoC.

that during the descent the battery is almost constantly recuperating energy, letting the $S o C$ slightly increase and the $H R$ decrease to a level of about $80 \mathrm{bpm}$. The very last ascent still requires some effort to the cyclist and quickly depletes the battery $S o C$ down to the target value.

Performance is summarized in Table 4 . Notice that in this case it is not possible to keep the $H R$ below the threshold $\widetilde{H R}$; the increase in the cumulated cost with policy $\pi_{s}$ is of $4 \%$ with respect to the optimum, while the peak $H R$ is $7 \mathrm{bpm}$ higher.

With the baseline strategy $\pi_{b}$, the profile of battery power $P_{b}$ is quite similar to the other strategies in the first segment (until minute 12); however, the level of assistance to the cyclist is slightly higher, as shown by the lower $H R$. As a consequence, the $S o C$ decreases more rapidly than with other strategies. In the following segment, during the long uphill, policy $\pi_{b}$ does not saturate the battery to avoid excessive depletion, therefore the load on the cy- 
Table 5

Real-time control with unknown elevation profile: simulation results on Track $\mathcal{T}_{3}$.

\begin{tabular}{ccccc}
\hline$S o C^{o}(T)(\%)$ & & $\Phi(H R)(\%)$ & $\|H R\|_{\infty}(\mathrm{bpm})$ & $\Gamma_{S o C}(\%)$ \\
\hline \multirow{3}{*}{35} & $\pi_{1}$ & 0 & 96 & 0 \\
& $\pi_{s}$ & 29 & 116 & 0 \\
& $\pi_{b}$ & 100 & 152 & 1 \\
\hline
\end{tabular}
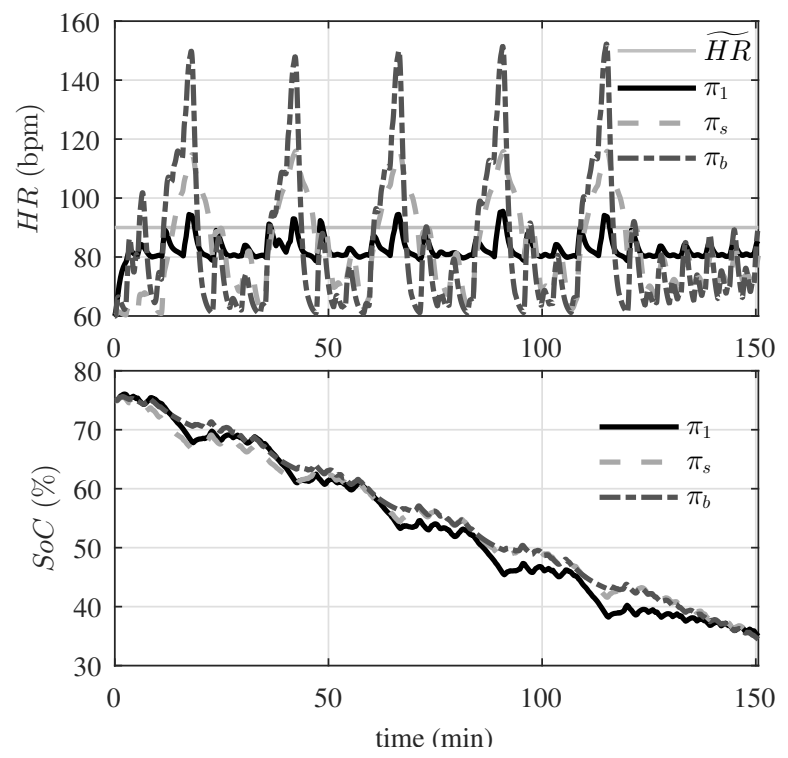

Fig. 10. Policies $\pi_{1}, \pi_{s}, \pi_{b}$ on Track $\mathcal{T}_{3}$ : heart rate $H R$ and state of charge $S o C$ when the elevation profile is unknown.

clist is very high. Notice that the peak value reached by the $H R$ (238 bpm) is unrealistic: in a real experiment, the cyclist would cut power generation and a less demanding speed profile would be realized.

Similar comments apply to the charge sustaining scenario $\left(S o C^{o}(T)=75 \%\right)$. By contrast, in the scenario of maximum assistance to the cyclist $\left(S o C^{o}(T)=67 \%\right)$ the performance of the three approaches is close.

This can be explained as follows: when the motor power $P_{m}$ is not negative - i.e. there is regenerative braking it is very often higher than the maximum battery power. As a consequence, the optimal strategy is, for most of the time, either to store braking energy or to deplete the battery at full power. In both cases, this is naturally done also by policy $\pi_{b}$, hence the close-to-optimum results.

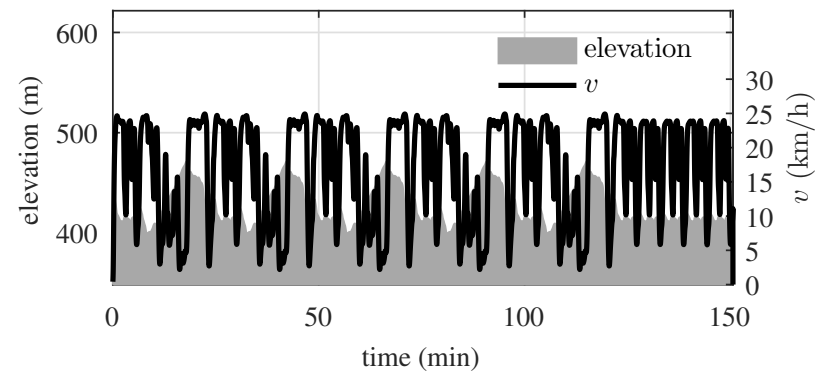

Fig. 11. Speed and elevation profiles on Track $\mathcal{T}_{3}$.
Table 6

Real-time control with known elevation profile: simulation results on Track $\mathcal{T}_{3}$.

\begin{tabular}{ccccc}
\hline$S o C^{o}(T)(\%)$ & & $\Phi(H R)(\%)$ & $\|H R\|_{\infty}(\mathrm{bpm})$ & $\Gamma_{S o C}(\%)$ \\
\hline \multirow{3}{*}{35} & $\pi_{1}$ & 1 & 96 & 0 \\
& $\pi_{s}$ & 2 & 96 & 0 \\
& $\pi_{b}$ & 100 & 115 & 0 \\
\hline
\end{tabular}
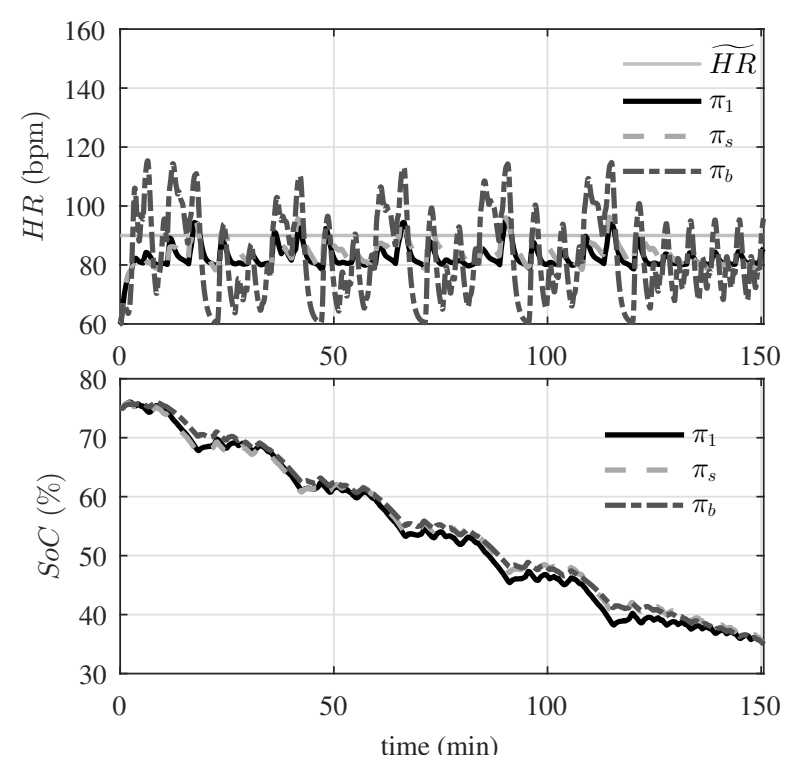

Fig. 12. Policies $\pi_{1}, \pi_{s}, \pi_{b}$ on Track $\mathcal{T}_{3}$ : heart rate $H R$ and state of charge $S o C$ when the elevation profile is known in advance.

\subsection{Sensitivity study on Cycling Track $\mathcal{T}_{3}$}

In this sub-section, we investigate to what extent the performance of policy $\pi_{s}$ is affected by the knowledge of the elevation profile. Policy $\pi_{b}$ actually does not use that information and generates the reference $S o C^{o}$ based on the trip length only.

To understand the effect of these different approaches, the two policies are here fed with the same reference $S o C^{o}$ : it is generated as explained in Section 5, first assuming that the track is flat, then considering also the elevation profile. Fig. 11 shows the speed and elevation profiles on track $\mathcal{T}_{3}$, which consists of 5 repetitions of a downhilluphill track followed by a brief, almost flat segment.

The results obtained when the elevation profile is not known in advance are shown in Fig. 10 and in Table 5. Having the same $S o C$ reference, policies $\pi_{s}$ and $\pi_{b}$ yield quite close $S o C$ trends. Both strategies fail to keep an almost constant $H R$ as policy $\pi_{1}$ does, because the suboptimal reference $S o C^{o}$ does not consider the presence of long uphills and downhills. The cyclist load and $H R$ are apparently influenced by the road profile; nonetheless, despite the reference $S o C^{o}$ is the same, the two policies have different power split strategies, and policy $\pi_{s}$ achieves a smoother $H R$ trajectory, lower cumulated cost (71\% less) and lower peak HR (36 bpm less).

The case when the elevation is known in advance and is used in the generation of the reference $S o C^{o}$ is depicted 
in Fig. 12 and in Table 6. The $S o C$ trends are closer to the optimal one than in the previous case. As for the $H R$, policy $\pi_{s}$ shows smaller and less frequent variations than the baseline policy $\pi_{b}$. This leads to a big difference in the cumulated cost (98\% less) and to a reduction of $19 \mathrm{bpm}$ of the peak $H R$.

\section{$7 \quad$ Experimental Results}

The experimental setup is described in Section 2. Fig. 13 depicts the speed profile tracked during the experiments: it is a real speed profile recorded on track $\mathcal{T}_{4}$, an urban path, for a duration of about $15 \mathrm{~min}$ and a distance of $3.3 \mathrm{~km}$, as summarized in Table 2. Tracking this speed profile using only battery power, i.e. letting $P_{g}=0$, would deplete about $7.5 \%$ of the $S o C$. The experiments are performed setting a target $S_{o} C^{\circ}(T)=S o C(0)-4.5 \%$ to reach at destination; in terms of battery charge usage, this represents an intermediate scenario between charge sustenance and maximum charge depletion, similar to those studied in simulation. We present here the results obtained in six experiments, all performed by one subject on the same bike equipped either with the proposed policy $\pi_{s}$ (in three tests) or with the baseline policy $\pi_{b}$ (in three other tests). Results from different tests under the same policy are shown in Fig. 13 with different lines of the same color and style.

The behavior of policy $\pi_{s}$ in the experiments is affected by the parameters of the cyclist model. For the experiments, policy $\pi_{s}$ is parametrized with the same values used so far, that were identified in [33] for a different subject. While this choice likely introduces uncertainty in the model, it also makes the experiments closer to a real application, because the identification procedure described in [33] is quite cumbersome and may not be repeated for every single user.

The results depicted in Fig. 13 and summarized in Table 7 suggest some observations. The trajectories of the battery power $P_{b}$, of the heart rate $H R$ and of the state of charge $S o C$ are pretty similar in the three different experiments, both with policy $\pi_{s}$ and with policy $\pi_{b}$. Clearly the $H R$ is subject to greater variations, that can be due also to other reasons than physical activity. When using policy $\pi_{s}$, variations in the $H R$ imply variations of the reference $P_{g}$ that is requested to the cyclist. Moreover, both with policy $\pi_{s}$ and with policy $\pi_{b}$, it is not guaranteed that the cyclist actually produces as much $P_{g}$ as requested. In closed loop, this mismatch justifies the small deviations in the trajectories of the $S o C$ and $P_{b}$ under the same policy.

Table 7

Real-time control: experimental results on Track $\mathcal{T}_{4}$.

\begin{tabular}{ccccc}
\hline$S o C^{o}(T)(\%)$ & & $\Phi(H R)(\%)$ & $\|H R\|_{\infty}(\mathrm{bpm})$ & $\Gamma_{\text {SoC }}(\%)$ \\
\hline \multirow{4}{*}{70.5} & & 29 & 107 & -5 \\
& $\pi_{s}$ & 31 & 106 & -2 \\
& & 31 & 109 & -3 \\
\cline { 2 - 5 } & & 59 & 130 & -4 \\
& $\pi_{b}$ & 62 & 137 & -4 \\
& 100 & 136 & -3 \\
\hline
\end{tabular}
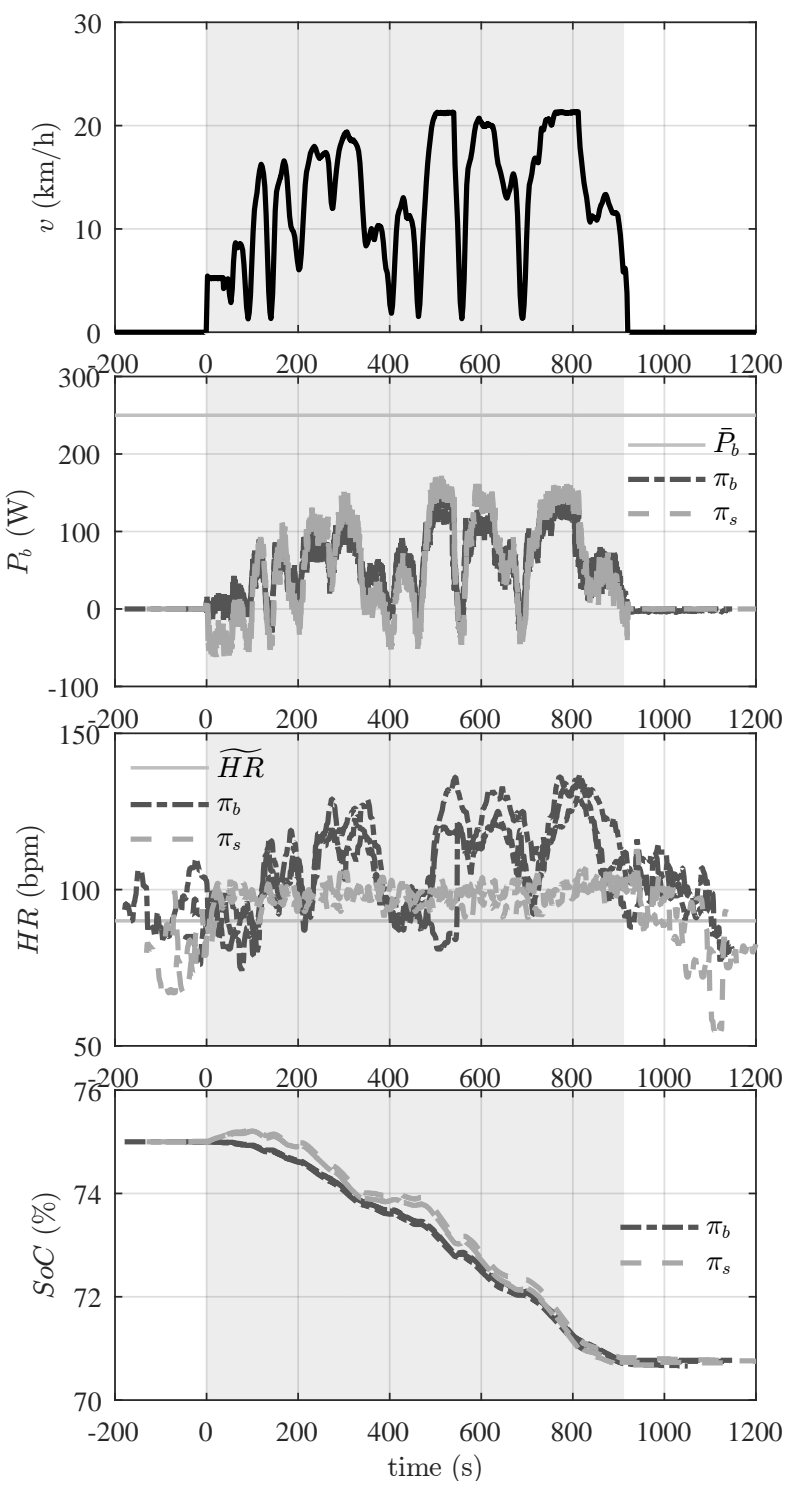

Fig. 13. Experimental validation of policies $\pi_{s}$ and $\pi_{b}$ on Track $\mathcal{T}_{4}$ : speed profile, battery power $P_{b}$, heart rate $H R$, state of charge $S o C$. Different lines of the same color and style refer to different tests performed using the same policy. The shaded areas highlight the segment when the experiment is ongoing.

In spite of small deviations, the experiments exhibit a behavior of the $H R$ similar to that observed in simulation. In particular, policy $\pi_{s}$ manages to keep the $H R$ approximately constant by properly adjusting the requested $P_{g}$. Policy $\pi_{b}$ instead causes large variations of the $H R$, that in two segments drops below the value obtained with policy $\pi_{s}$, but for most of the time is significantly higher. These apparent differences in the $H R$ trajectories are reflected in less apparent differences in the $P_{b}$ and $S o C$ trajectories. Nonetheless, the target $S o C^{O}$ is met by both policies in all the experiments, as can be inferred both from Fig. 13 and from the last column in Table 7.

As for the global performance, summarized in Table 7, the proposed policy $\pi_{s}$ outperforms the baseline policy $\pi_{b}$, both in terms of cumulated cost and in terms of peak $H R$. A possible direction for future research is the adap- 

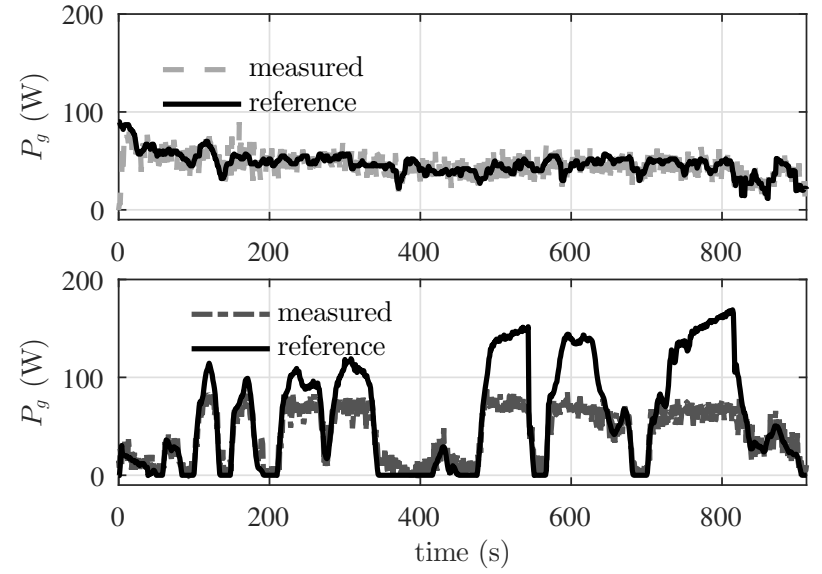

Fig. 14. Experimental validation of policies $\pi_{s}$ (top) and $\pi_{b}$ (bottom) on Track $\mathcal{T}_{4}$ : reference and measured $P_{g}$.

tation to human variations; notice, however, that human model uncertainty is not only due to differences between subjects, but also to disregarded physiological and psychological factors.

Table 7 shows that the indexes of policy $\pi_{b}$ are subject to a greater variability than those of policy $\pi_{s}$. Fig. 14, in which the reference and measured $P_{g}$ are compared, provides some insight into this. It can be observed that the reference is well tracked, apart from some fluctuations at relatively high frequency (that are due to the non-uniform supply of torque during a pedal revolution). However, in some segments policy $\pi_{b}$ requires high power generation and the cyclist fails to track it. While the excess power is supplied by the battery, the global performance summarized in Table 7 appears to be affected by the behavior during these segments, that changes quite unpredictably from test to test. This is unavoidable, regardless of the employed strategy.

\section{Conclusion}

This paper deals with real-time energy management of a series HEB. The main goals of the supervisor are to minimize the exertion perceived by the cyclist and to guarantee that the battery $S o C$ at the end of a trip approaches a target defined by the cyclist. An approximated solution to the acausal OCP is used to develop a real-time algorithm. The use of route information is found to be quite important to ensure that the battery $S o C$ at the end of the trip is close to the target set by the user; this in turn affects the global performance in terms of perceived exertion. Results from simulations and experiments are discussed to evaluate the performance of the proposed realtime framework, also in the presence of uncertain route information. Possible future work includes the extension of the approach to parallel HEBs.

\section{References}

[1] J. Guanetti, S. Formentin, M. Corno, S. M. Savaresi, Optimal energy management in series hybrid electric bicycles, in: IEEE 54th Conference on Decision and Control, 2015, pp. 869-874.
[2] G. Alli, S. Formentin, S. M. Savaresi, On the Suitability of EPACs in urban use, in: 5th IFAC Symposium on Mechatronic Systems, 2010.

[3] T. van Keulen, J. Gillot, B. de Jager, M. Steinbuch, Solution for state constrained optimal control problems applied to power split control for hybrid vehicles, Automatica 50 (1) (2013) 187-192.

[4] B. Sampathnarayanan, S. Onori, S. Yurkovich, An optimal regulation strategy with disturbance rejection for energy management of hybrid electric vehicles, Automatica 50 (1) (2014) 128-140.

[5] A. Sciarretta, L. Guzzella, Control of Hybrid Electric Vehicles, IEEE Control Systems Magazine (2) (2007) 60-70.

[6] L. Guzzella, A. Sciarretta, Vehicle Propulsion Systems, Springer, 2007.

[7] L. Serrao, S. Onori, G. Rizzoni, ECMS as a realization of Pontryagin's Minimum Principle for HEV control, in: American Control Conference, 2009, pp. 3964-3969.

[8] A. Sciarretta, L. Guzzella, M. Back, A real-time optimal control strategy for parallel hybrid vehicles with on-board estimation of the control parameters, in: IFAC symposium on advances in automotive control, 2004, pp. 502-507.

[9] C. Musardo, G. Rizzoni, Y. Guezennec, B. Staccia, A-ECMS: An Adaptive Algorithm for Hybrid Electric Vehicle Energy Management, European Journal of Control 11 (4-5) (2005) 509-524.

[10] A. Sciarretta, L. Serrao, P. Dewangan, P. Tona, E. Bergshoeff, C. Bordons, L. Charmpa, P. Elbert, L. Eriksson, T. Hofman, M. Hubacher, P. Isenegger, F. Lacandia, A. Laveau, H. Li, D. Marcos, T. Nüesch, S. Onori, P. Pisu, J. Rios, E. Silvas, M. Sivertsson, L. Tribioli, A.-J. van der Hoeven, M. Wu, A control benchmark on the energy management of a plug-in hybrid electric vehicle, Control Engineering Practice 29 (2014) 287-298.

[11] C. Manzie, P. Dewangan, G. Corde, O. Grondin, A. Sciarretta, State of Charge Management for Plug-In Hybrid Vehicles With Uncertain Trip Information, Journal of Dynamic Systems, Measurement, and Control 137 (9) (2015) 091005.

[12] V. Larsson, Route Optimized Energy Management of Plug-in Hybrid Electric Vehicles, Ph.D. thesis, Chalmers University of Technology (2014).

[13] D. Ambühl, L. Guzzella, Predictive Reference Signal Generator for Hybrid Electric Vehicles, IEEE Transactions on Vehicular Technology 58 (9) (2009) 4730-4740.

[14] S. A. Fayazi, S. Lucich, A. Vahidi, G. Mocko, Optimal pacing in a cycling time-trial considering cyclist's fatigue dynamics, in: American Control Conference, 2013, pp. 6442-6447.

[15] N. Wan, S. A. Fayazi, H. Saeidi, A. Vahidi, Optimal power management of an electric bicycle based on terrain preview and considering human fatigue dynamics, in: American Control Conference, 2014, pp. 3462-3467.

[16] P. Spagnol, G. Alli, C. Spelta, P. Lisanti, F. Todeschini, S. M Savaresi, A. Morelli, A full hybrid electric bike: How to increase human efficiency, in: American Control Conference, 2012, pp. 2761-2766.

[17] P. Spagnol, M. Corno, S. M. Savaresi, Pedaling torque reconstruction for half pedaling sensor, in: European Control Conference, 2013, pp. 275-280.

[18] P. Spagnol, M. Corno, R. Mura, S. M. Savaresi, Self-sustaining strategy for a hybrid electric bike, in: American Control Conference, 2013, pp. 3479-3484.

[19] Mando Footloose, http://www . mandofootloose.com/.

[20] A. Fuchs, Series hybrid pedelecs and the electronic pedal, ExtraEnergy Pedelec and E-Bike Magazine (2015) 42-47.

[21] M. Corno, D. Berretta, S. M. Savaresi, Human Machine Interfacing Issues in SeNZA, a Series Hybrid Electric Bicycle, in: American Control Conference, 2015, pp. 1149-1154. 
[22] M. Corno, F. Roselli, S. M. Savaresi, Bilateral Control of SeNZA - A Series Hybrid Electric Bicycle, IEEE Transactions on Control Systems Technology (Available online).

[23] A. V. Rao, A survey of numerical methods for optimal control, Advances in the Astronautical Sciences 135 (1) (2009) 497528

[24] P. Spagnol, S. Rossi, S. M. Savaresi, Kalman filter SOC estimation for Li-ion batteries, in: IEEE International Conference on Control Applications, 2011, pp. 587-592.

[25] J. Chavarren, J. Calbet, Cycling efficiency and pedalling frequency in road cyclists, European journal of applied physiology and occupational physiology 80 (6) (1999) 555-63.

[26] A. Belli, F. Hintzy, Influence of pedalling rate on the energy cost of cycling in humans, European journal of applied physiology 88 (1-2) (2002) 158-62.

[27] O. i. Foss, J. Hallén, Cadence and performance in elite cyclists, European journal of applied physiology 93 (4) (2005) 453-62.

[28] U. Emanuele, J. Denoth, Power-cadence relationship in endurance cycling, European journal of applied physiology 112 (1) (2012) 365-75.

[29] S. Annaheim, U. Boutellier, G. Kohler, The energetically optimal cadence decreases after prolonged cycling exercise, European journal of applied physiology 109 (6) (2010) 110310 .

[30] R. H. Morton, D. J. Hodgson, The relationship between power output and endurance: a brief review, European journal of applied physiology and occupational physiology 73 (6) (1996) 491-502.

[31] T. Dahmen, D. Saupe, S. Wolf, Applications of mathematical models of road cycling, in: 7th Conference on Mathematical Modeling, 2012, pp. 804-809.

[32] G. Borg, P. Hassmn, M. Lagerstrm, Perceived exertion related to heart rate and blood lactate during arm and leg exercise, European Journal of Applied Physiology 65 (85) (1987) 679685

[33] M. Corno, P. Giani, M. Tanelli, S. M. Savaresi, Human-in-theLoop Bicycle Control via Active Heart Rate Regulation, IEEE Transactions on Control Systems Technology (2014) 1-12.

[34] S. Formentin, J. Guanetti, S. M. Savaresi, Least costly energy management for series hybrid electric vehicles, Control Engineering Practice 48 (2016) 37-51.

[35] J. Guanetti, S. Formentin, S. M. Savaresi, Energy management system for an electric vehicle with a rental range extender: A least costly approach, IEEE Transactions on Intelligent Transportation Systems 17 (11) (2016) 3022-3034.

[36] J. Guanetti, S. Formentin, S. M. Savaresi, Least costly energy management for electric vehicles with plug-in range extenders, in: IEEE 54th Conference on Decision and Control, 2015, pp. 638-643.

[37] J. Guanetti, S. Formentin, S. M. Savaresi, Total cost minimization for next generation hybrid electric vehicles, in: 19th IFAC World Congress, 2014, pp. 4819-4824.

[38] S. Onori, L. Serrao, G. Rizzoni, Adaptive equivalent consumption minimization strategy for hybrid electric vehicles, in: ASME 2010 dynamic systems and control conference, American Society of Mechanical Engineers, 2010, pp. $499-505$.

[39] M. Sivertsson, C. Sundström, L. Eriksson, Adaptive control of a hybrid powertrain with map-based ECMS, IFAC Proceedings Volumes 44 (1) (2011) 2949-2954.

[40] M. Sivertsson, Adaptive control using map-based ECMS for a PHEV, IFAC Proceedings Volumes 45 (30) (2012) 357-362. 